\title{
Identification of differentially expressed genes associated with burn sepsis using microarray
}

\author{
XIAOLI XU ${ }^{1}$, ZHAORONG SHI $^{2}$, JIALE HU ${ }^{2}$, BO YUAN ${ }^{2}$, HUIMIN HUANG $^{1}$, HONGMEI FANG $^{1}$, \\ XIANGYI YIN $^{1}$, NIUYAN NIE ${ }^{1}$ and XIAOYUE SHENG ${ }^{1}$ \\ Departments of ${ }^{1}$ Infection Management and ${ }^{2}$ Medical Administration, Jinling Hospital, \\ Medical School of Nanjing University, Nanjing, Jiangsu 210002, P.R. China
}

Received February 2, 2015; Accepted August 20, 2015

DOI: $10.3892 /$ ijmm.2015.2374

\begin{abstract}
The aim of the present study was to identify the potential target biomarkers associated with burn sepsis using microarray. GSE1781 was downloaded from Gene Expression Omnibus and included a collective of three biological replicates for each of the three conditions: Sham-Sham, Sham-cecal ligation and puncture (CLP) and Burn-CLP. Subsequently, limma was applied to screen the differentially expressed genes (DEGs). Additionally, functional annotations were predicted by pathway enrichment. Furthermore, the transcription factors were screened according to the transcriptional regulation from patterns to profiles database. Furthermore, the interaction associations of the proteins were obtained from the STRING database and the protein-protein interaction (PPI) network was constructed using Cytoscape. Finally, the gene co-expression analysis was conducted using CoExpress. In total, compared with Sham-Sham, a total of 476 DEGs and 682 DEGs were obtained in Sham-CLP and Burn-CLP, respectively. Additionally, 230 DEGs were screened in Burn-CLP compared with Sham-CLP. Acadm, Ehhadh and Angptl4 were significantly enriched in the PPAR signaling pathway. Additionally, Gsta3, Gstm2 and Gstt1 in Burn-CLP were significantly enriched in glutathione metabolism. In the PPI network, the transcription factor Ppargcla interacted with Angptl4, while Acadm interacted with Ehhadh. The gene co-expression analysis showed that Ehhadh could be co-expressed with Aqp8. In conclusion, Acadm, Ehhadh, Aqp8, Gsta3, Gstm2, Gstt1, Ppargcla and Angptl4 may be potential target genes for the treatment of burn sepsis.
\end{abstract}

Correspondence to: $\mathrm{Dr}$ Xiaoli $\mathrm{Xu}$, Department of Infection Management, Jinling Hospital, Medical School of Nanjing University, 305 East Zhongshan Road, Nanjing, Jiangsu 210002, P.R. China

E-mail: xiaolixuxxx@163.com

Dr Zhaorong Shi, Department of Medical Administration, Jinling Hospital, Medical School of Nanjing University, 305 East Zhongshan Road, Nanjing, Jiangsu 210002, P.R. China

E-mail: zhaorongshshh@126.com

Key words: burn sepsis, functional enrichment, genes co-expression

\section{Introduction}

Sepsis, which is a systemic inflammatory syndrome, is triggered by severe infections (1). Infection is the most common and serious complication for the patients with a major burn (2). Once the burn wound is infected by the bacteria, bacterium could rapidly proliferate within the damaged tissue, which leads to sepsis and septic shock (3). Previously, it was reported that $50-60 \%$ of burn patients with sepsis succumb to the condition (4). Therefore, it is of great urgency to establish the mechanism of burn sepsis.

Previously, a study by Beffa et al (4) indicated that the interleukin 6 levels in burn mice were significantly increased by cecal ligation and puncture (CLP). However, the interleukin 6 levels did not decrease in the recovery patients following treatment with statin showing that there should be other mechanisms of sepsis in the burn patients. Additionally, high levels of interleukin 8 are correlated with increased multi-organ failure, sepsis and mortality in post-burn patients (5). Additionally, intestinal regulatory $\mathrm{T}$ cell expression has been found to exert immunosuppressive effects on other intestinal $\mathrm{T}$ lymphocytes and be closely associated with endotoxin translocation in porcine sepsis following severe burns injuries (6). However, the molecular mechanism of burn sepsis remains unclear.

In 2007, Banta et al (7) used moderate burn injury followed by CLP (used for producing sepsis) to construct three groups of rats: Sham Burn-Sham CLP (Sham-Sham), Sham Burn-CLP (Sham-CLP) and Burn-CLP. Subsequently, a microarray expression profile was conducted to screen the differentially expressed genes with the methods of significance analysis of microarrays and false discovery rate of $10 \%$ to investigate the contribution of gene expression to metabolic fluxes in hypermetabolic livers induced by burn injury and CLP in rats and identified that burn injury combined with CLP led to the most significant changes, while CLP alone significantly increased metabolic gene expression; however, it decreased a number of the corresponding metabolic fluxes.

The present study aimed to use the same microarray data to further screen the DEGs between Sham-CLP and Sham-Sham, Burn-CLP and Sham-Sham, as well as Burn-CLP and Sham-CLP with the limma package based on the criteria of $\mathrm{P}<0.05$ and $\mid \log _{2}$ fold change (FC) $\mid \geq 2$ and collected the specific genes associated with burn sepsis. Additionally, the 
Kyoto Encyclopedia of Genes and Genomes (KEGG) pathway enrichment, transcription factor screening, protein-protein interaction (PPI) network construction and co-expression analysis of DEGs were also conducted to illustrate the mechanism of burn sepsis. A previous study proposed that analysis based on differential statistical tests may result in different outcomes (8). Therefore, we hypothesized that certain different results may be obtained from the data of Banta et al (7).

\section{Materials and methods}

Microarray data. The expression profile of GSE1781 deposited by Banta et al (7) was downloaded from Gene Expression Omnibus (GEO; http://www.ncbi.nlm.nih.gov/geo/), which was based on the platform of the GPL341 [RAE230A] Affymetrix Rat Expression 230A array. GSE1781 included a collection of three biological replicates for each of the three conditions: Sham-Sham, Sham-CLP and Burn-CLP.

Data preprocessing and DEGs screening. The downloaded data were normalized using preprocess Core (9). The probes, which were not mapped to the corresponding gene symbols, were abandoned. Furthermore, the average expression value was used for the genes corresponding to multiple probes. Subsequently, the limma (linear models for microarray data) package in R/Bioconductor was employed to screen the DEGs between Sham-CLP and Sham-Sham, Burn-CLP and Sham-Sham, as well as Burn-CLP and Sham-CLP. $P<0.05$ and $\mid \log _{2} \mathrm{FCl} \geq 2$ were used as the cut-off criteria for the DEGs.

Pathway enrichment analysis. The Database for Annotation, Visualization and Integrated Discovery is a collection of functional annotation tools for investigators to study the biological meaning behind a large list of genes (10). The KEGG pathway database (http://www.genome.jp/kegg/pathway.html), which includes the functions in terms of the network of the interacting molecules, was used to perform the pathway enrichment for the DEGs (11). $\mathrm{P}<0.05$ was the threshold for the pathway enrichment analysis.

Transcription factors screening. The transcriptional regulation from patterns to profiles (TRANSFAC) database (http://www. gene-regulation.com) containing the data on transcription factors, their target genes and regulatory binding sites was applied to discover the transcription factors (12). Additionally, different transcription factors between the Sham-CLP and Burn-CLP were further analyzed.

PPI network construction. The interaction associations of the proteins were analyzed using the online tool Search Tool for the Retrieval of Interacting Genes (STRING; $\mathrm{http} / / /$ string-db.org/)(13) and the required confidence (combined score) $\geq 0.4$ was used as the cut-off criterion. Subsequently, Cytoscape was used to visualize the network (14).

Co-expression analysis of DEGs in Burn-CLP compared with Sham-Sham and Sham-CLP. The union of DEGs in Burn-CLP and Sham-Sham, as well as the DEGs in Burn-CLP and Sham-CLP, was screened. Subsequently, CoExpress (http://www.bioinformatics.lu/CoExpress/) was employed to calculate the correlation coefficient of the DEGs. Pearson correlation coefficient was used to reflect the expression correlation between the DEGs. The Pearson correlation coefficient $>0.9$ was taken as the threshold.

\section{Results}

DEG analysis. Compared with Sham-Sham, a total of 476 DEGs (including 225 upregulated DEGs, such as Lcn2 and Zfhx2, and 251 downregulated DEGs, such as Tnnt1 and $S v 2 a$ ) and 682 DEGs (including 324 upregulated DEGs, such as Arl6ip6 and Pla2g2a, and 358 downregulated DEGs, such as Acadm and Ehhadh) were obtained in Sham-CLP and Burn-CLP, respectively. Additionally, 230 DEGs, including 85 upregulated DEGs, such as Rbbp9 and Clca4, and 145 downregulated DEGs, such as Igfals and GOs2, were screened in Burn-CLP compared with Sham-CLP. The 10 most significantly upregulated and downregulated DEGs are listed in Table I. Additionally, the hierarchical cluster analysis is shown in Fig. 1.

KEGG pathway enrichment. Compared with Sham-Sham, the upregulated DEGs, such as Cxcl14, Cxcll6 and Cxcr4, in Burn-CLP were significantly enriched in the chemokine-signaling pathway $(\mathrm{P}=0.015)$ (Table II). The downregulated DEGs, such as Acadm and Ehhadh, were significantly enriched in the PPAR signaling pathway $\left(\mathrm{P}=8.298 \times 10^{-4}\right)$. Additionally, Gsta3, Gstm2 and Gstt1 in Burn-CLP were significantly enriched in glutathione metabolism $(\mathrm{P}=0.023)$ (Table III).

Transcription factor analysis. According to the TRANSFAC database, a total of 18 (including Ascl2 and Atf3) and 19 (including Ascl2 and Ppargcla) transcription factors were screened among the DEGs in Sham-CLP and Burn-CLP, respectively, compared with Sham-Sham. Additionally, Sham-CLP and Burn-CLP possessed 8 identical transcription factors (Fig. 2A). Their expression levels are exhibited in Fig. 2. Apart from these 8 transcription factors, there were 11 transcription factors (including Ppargcla and $D B P$ ) in Burn-CLP compared with Sham-Sham (Fig. 2B).

PPI network analysis. The PPI network for the DEGs in the three comparison groups: Sham-Sham versus Sham-CLP, Sham-Sham versus Burn-CLP. Burn-CLP versus Sham-CLP, are shown in Fig. 3C. For the DEGs between Sham-Sham and Sham-CLP, a total of 361 pairs of PPI were obtained from the STRING database. In the PPI network, Tnf possessed the highest degree of 25 (Fig. 3A). Additionally, for the DEGs between Sham-Sham and Burn-CLP, a total of 595 pairs of PPI were obtained. In the PPI network, Esrl had the highest degree of 31 and Ppargcla could interact with Angptl4 (Fig. 3B). As for the DEGs between Burn-CLP and Sham-CLP, a total of 110 pairs of PPI were obtained. In the PPI network, Pik3rl had the highest degree of 9 (Fig. 3C).

Co-expression analysis. A total of 413 pairs of co-expression associations, including 105 genes, were obtained (Fig. 4). Among these genes, Cyp $3 a 9$ could be co-expressed with 13 genes (such as Atplbl and Ell2). In addition, Ehhadh could be co-expressed with Aldh3a2, Aqp8 and Bdhl. 
Table I. Ten most significantly upregulated and downregulated DEGs in Sham-CLP and Burn-CLP compared with Sham-Sham and the DEGs in Burn-CLP compared with Sham-CLP.

\begin{tabular}{|c|c|c|c|c|c|c|c|c|c|}
\hline & \multicolumn{3}{|c|}{ Sham-CLP vs. Sham-Sham } & \multicolumn{3}{|c|}{ Burn-CLP vs. Sham-Sham } & \multicolumn{3}{|c|}{ Burn-CLP vs. Sham-CLP } \\
\hline & DEGs & $\log \mathrm{FC}$ & P-value & DEGs & $\log \mathrm{FC}$ & P-value & DEGs & $\log \mathrm{FC}$ & P-value \\
\hline \multirow[t]{10}{*}{ Upregulated } & Lcn2 & 4.602548 & $3.83 \times 10^{-8}$ & Arl6ip6 & 5.066561 & 0.008025 & Rbbp9 & 3.296154 & 0.027772 \\
\hline & $Z f h x 2$ & 4.02276 & 0.000262 & Pla2g $2 a$ & 4.675226 & $5.34 \times 10^{-7}$ & Clca4 & 3.295737 & $1.43 \times 10^{-5}$ \\
\hline & Pla2g2a & 3.445816 & $5.64 \times 10^{-6}$ & Lcn2 & 4.596656 & $3.86 \times 10^{-8}$ & Tafl & 2.915533 & 0.023838 \\
\hline & Plscr2 & 3.371389 & 0.037682 & $\mathrm{Neb}$ & 4.304198 & 0.000744 & Tnnt1 & 2.829013 & $6.28 \times 10^{-5}$ \\
\hline & $\mathrm{Neb}$ & 3.345386 & 0.003449 & Zfhx2 & 4.267401 & 0.000174 & Ppargcla & 2.749841 & 0.036676 \\
\hline & $\operatorname{Itg} b 3 b p$ & 3.148196 & 0.005818 & S100a9 & 3.878773 & 0.000332 & Stard7 & 2.74522 & 0.004186 \\
\hline & $N f y b$ & 3.076118 & 0.006189 & Plscr2 & 3.500379 & 0.032474 & Pitx2 & 2.567246 & 0.015435 \\
\hline & Eif4e3 & 3.026708 & 0.004705 & Ly49s7 & 3.315606 & $9.63 \times 10^{-5}$ & Smim3 & 2.395974 & 0.005767 \\
\hline & Mmrnl & 3.011887 & 0.010381 & Ddit4 & 3.230954 & 0.009239 & Aurka & 2.347335 & 0.002993 \\
\hline & Sall2 & 2.919577 & 0.03118 & Sall2 & 3.217558 & 0.020624 & Rnfl13al & 2.168197 & 0.011317 \\
\hline \multirow[t]{10}{*}{ Downregulated } & Tnnt1 & -4.03526 & $4.48 \times 10^{-6}$ & $G 0 s 2$ & -5.3812 & 0.005036 & GOs2 & -5.50634 & 0.004437 \\
\hline & $S v 2 a$ & -3.9502 & 0.001074 & Igfals & -4.95741 & 0.001784 & Igfals & -4.2644 & 0.004283 \\
\hline & Pitx2 & -3.73666 & 0.002074 & $B d h 1$ & -4.40114 & 0.009124 & $B d h 1$ & -3.23361 & 0.036533 \\
\hline & $K l k 1$ & -3.43142 & 0.00292 & $H s d 17 b 2$ & -4.07748 & 0.001695 & Tpgs 1 & -2.91959 & 0.000561 \\
\hline & Scgbld2 & -3.30923 & 0.000102 & Niml & -3.73986 & 0.005966 & Tmedl & -2.74594 & $8.49 \times 10^{-6}$ \\
\hline & Rbbp9 & -3.25999 & 0.029075 & Car3 & -3.69018 & 0.000853 & Rnf213 & -2.64719 & 0.002884 \\
\hline & LOC691984 & -3.22479 & 0.017167 & Nrep & -3.53501 & 0.000151 & S1pr3 & -2.6437 & 0.010497 \\
\hline & Kcnjl & -3.19046 & 0.003682 & Lspl & -3.15146 & 0.00084 & Car3 & -2.64229 & 0.006101 \\
\hline & Gpr85 & -3.16711 & 0.000145 & Fabp3 & -3.10453 & 0.000821 & Gpr108 & -2.62186 & 0.012436 \\
\hline & Atpla4 & -3.12916 & 0.015975 & Apol9a & -3.09277 & 0.000172 & $O b f c l$ & -2.56692 & 0.001367 \\
\hline
\end{tabular}

DEGs, differentially expressed genes; CLP, cecal ligation and puncture; FC, fold change.

A
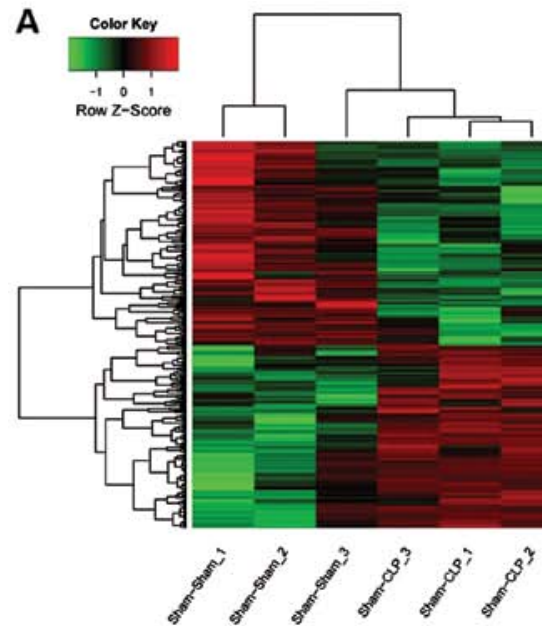

B

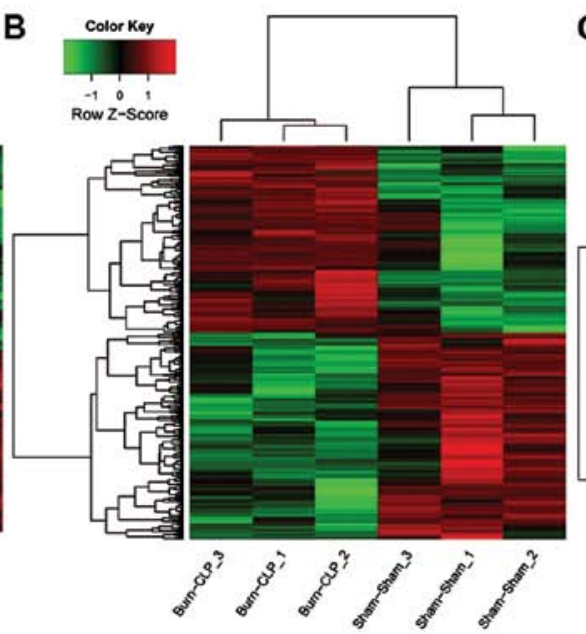

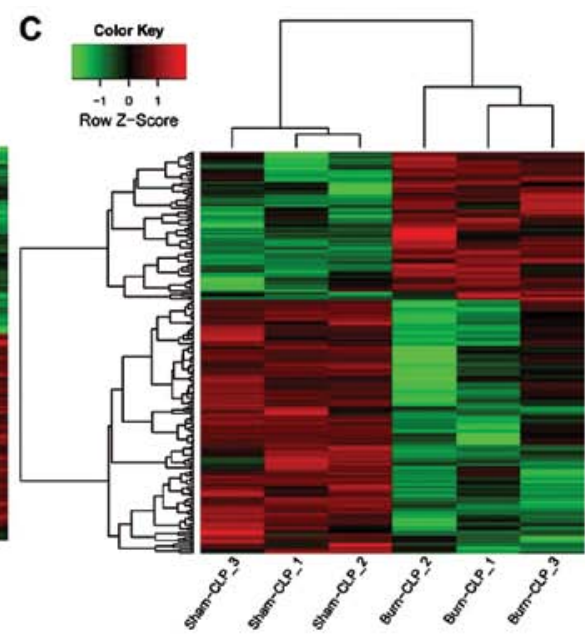

Figure 1. Hierarchical clusters of the DEGs in the three comparison groups. Hierarchical clusters of the DEGs between (A) Sham-Sham and Sham-CLP, (B) Sham-Sham and Burn-CLP and (C) Burn-CLP and Sham-CLP. DEGs, differentially expressed genes.

\section{Discussion}

In the present study, 682 DEGs were screened in Burn-CLP compared with Sham-Sham. The downregulated DEGs, such as Acadm and Ehhadh, were significantly enriched in PPAR signaling pathway. Additionally, in the PPI network, Acadm could interact with Ehhadh. A former study identified that the inhibition of PPAR $\gamma$ could possibly act as protection against T-cell death, which improved the defense mechanisms during systemic inflammation and sepsis (15). Apart from the aforementioned function, PPAR $\gamma$ could also be involved in the regulation of the mitochondrial dysfunction with tumor necrosis factor $\alpha$ (16). A study by Singer (17) illustrated that mitochondrial dysfunction could give rise to sepsis. As for 
Table II. KEGG pathway enrichments for the upregulated DEGs in the Sham-CLP and Burn-CLP compared with Sham-Sham.

\begin{tabular}{|c|c|c|c|}
\hline Groups & KEGG & P-value & Gene symbol \\
\hline \multirow[t]{3}{*}{ Sham-CLP vs. Sham-Sham } & rno00900: Terpenoid backbone biosynthesis & $3.85 \times 10^{-5}$ & Acat2, Fdps, Hmgcr, Idi1, Mvd \\
\hline & rno00100: Steroid biosynthesis & $8.85 \times 10^{-5}$ & Cyp51, Ebp, Hsd17b7, Lss, Sqle \\
\hline & rno04520: Adherens junction & 0.004 & $\begin{array}{l}\text { Acvrlc, Crebbp, Csnk2al, Ptpn1, } \\
\text { Ptprj, Ssx2ip }\end{array}$ \\
\hline \multirow[t]{3}{*}{ Burn-CLP vs. Sham-Sham } & rno00830: Retinol metabolism & 0.005 & $\begin{array}{l}\text { Cypla1, Cyp26a1, Cyp2b12, } \\
\text { Cyp3a9, Dhrs9, RGD1562200 }\end{array}$ \\
\hline & $\begin{array}{l}\text { rno04060: Cytokine-cytokine } \\
\text { receptor interaction }\end{array}$ & 0.012 & $\begin{array}{l}\text { Amhr2, Ccr1, Csf1r, Cxcl14, } \\
\text { Cxcl16, Cxcr4, Il1rap, Lifr, } \\
\text { Pf4, Tnfrsf } 21\end{array}$ \\
\hline & rno04062: Chemokine signaling pathway & 0.015 & $\begin{array}{l}\text { Adcy3, Ccr1, Cxcl14, Cxcl16, } \\
\text { Cxcr4, Grk5, Pf4, Rock1, Wasl }\end{array}$ \\
\hline
\end{tabular}

DEGs, differentially expressed genes; CLP, cecum ligation and puncture.
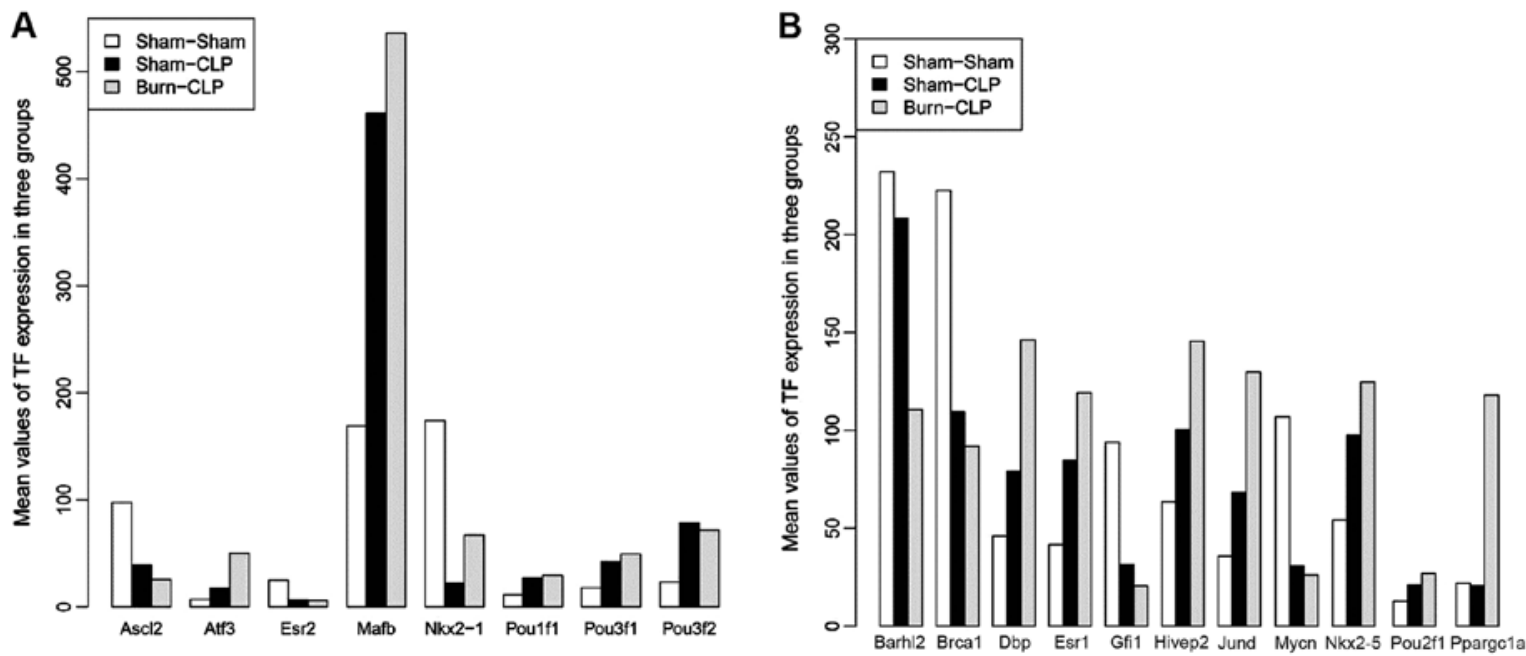

Figure 2. Significantly differentially expressed transcription factors in Sham-CLP and Burn-CLP compared with Sham-Sham. (A) The 8 significantly differentially expressed transcription factors in Sham-CLP and Burn-CLP compared with Sham-Sham. (B) The 11 significantly differentially expressed transcription factors only in Burn-CLP compared with Sham-Sham. CLP, cecum ligation and puncture.

the DEGs enriched in this pathway, Acadm, a member of the acyl-CoA dehydrogenase (ACAD) family, has been found to be involved in the metabolism of medium-chain fatty acids (18). In addition, Ehhadh has also been proven to be an indispensable element for the production of medium-chain dicarboxylic acids (19). In 2013, Hecker et al (20) obtained the conclusion that medium-chain fatty acids could serve as energy for the mitochondrial respiratory capacity and inflammatory conditions in sepsis. Therefore, we hypothesized that the interaction of Acadm and Ehhadh could be associated with sepsis by modulating the mitochondrial function through the PPAR signaling pathway.

Furthermore, the gene co-expression analysis showed that Ehhadh could be co-expressed with Aqp8. Aqp8 is a water channel protein on the inner mitochondrial membrane (21). The upregulation of Aqp 8 has been found to protect the mitochondria from damage in sepsis, which could lead to the loss of energy (22). Additionally, it has been discovered that Aqp8 was involved in $\mathrm{H}_{2} \mathrm{O}_{2}$ release and decrease of reactive oxygen species (ROS) production, which could damage the cells and antioxidant defense system and thus lead to sepsis $(23,24)$. Ehhadh has been found to be involved in mitochondrial fatty acid $\beta$-oxidation (25). Therefore, we hypothesized that the co-expression of Ehhadh and Aqp 8 could be associated with sepsis by regulating the mitochondrial function.

Furthermore, compared with Sham-Sham, the downregulated DEGs, Gsta3, Gstm2 and Gstt1, in Burn-CLP were significantly enriched in glutathione metabolism. The PPI network showed that interactions existed among these three proteins. Previous studies have shown that improved outcomes in animal models of sepsis were obtained by utilizing mitochondrial-targeted antioxidants $(26,27)$. Glutathione could protect the mitochondria from dysfunction by the detoxification of hydrogen peroxide in sepsis (28). Gsta3, Gstm2 and Gsttl encode the glutathione S-transferase $\alpha 3$, glutathione S-transferase $\mu 2$ and glutathione $\mathrm{S}$-transferase $\theta 1$, respectively. Glutathione S-transferases are well known for removing endogenous toxic compounds through glutathionylation of 
A

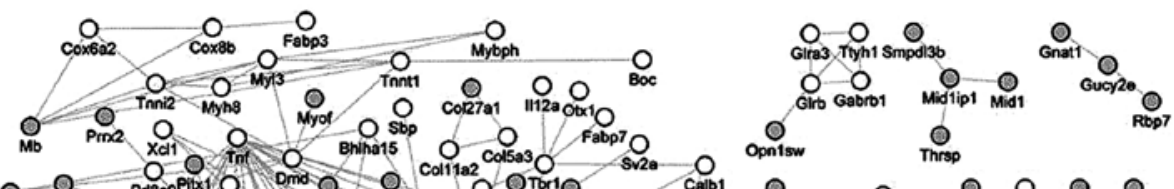

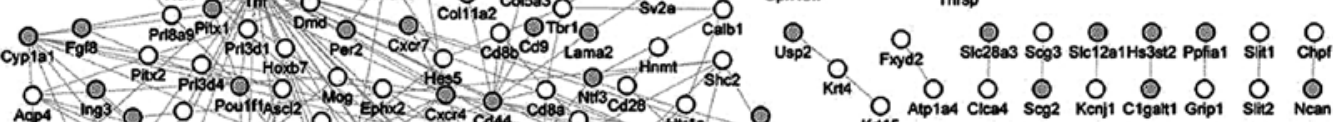

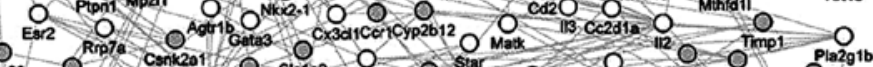

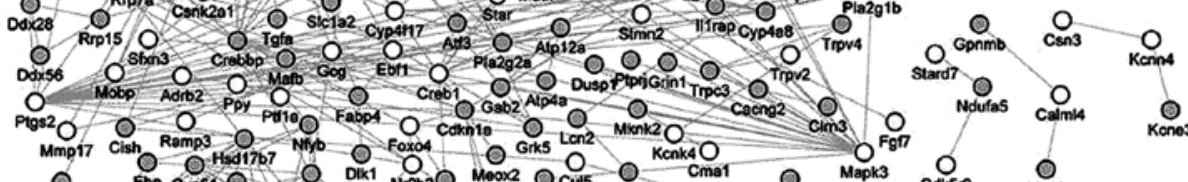

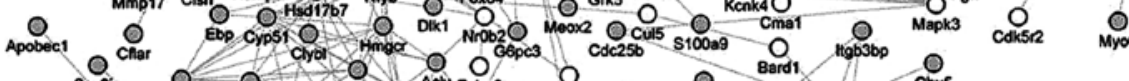

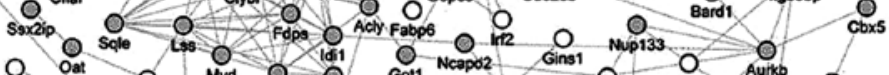

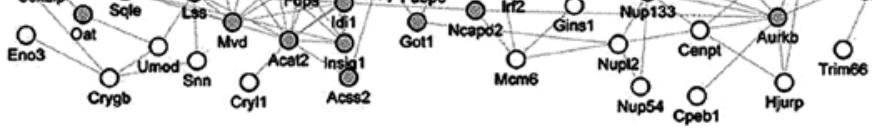

B

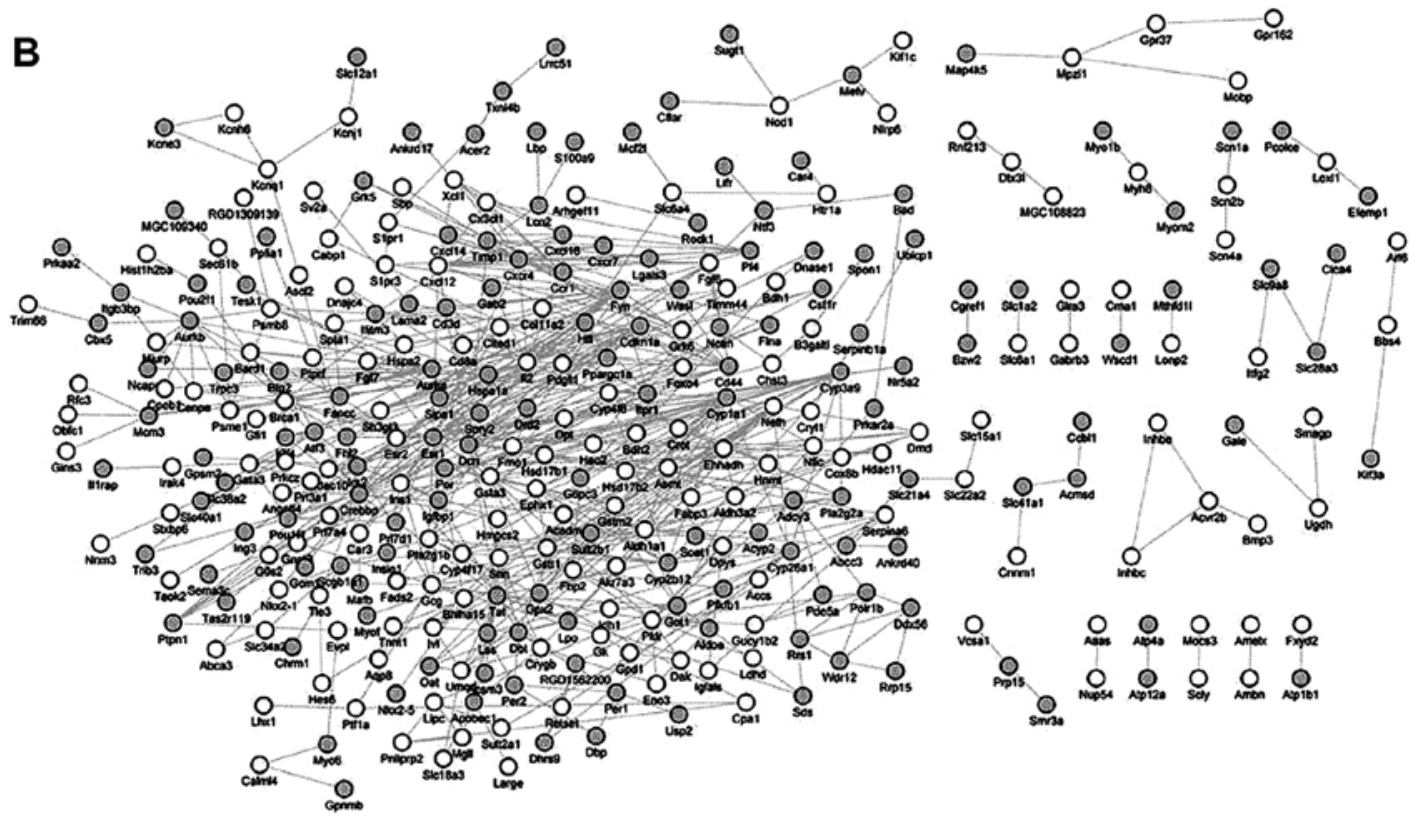

C

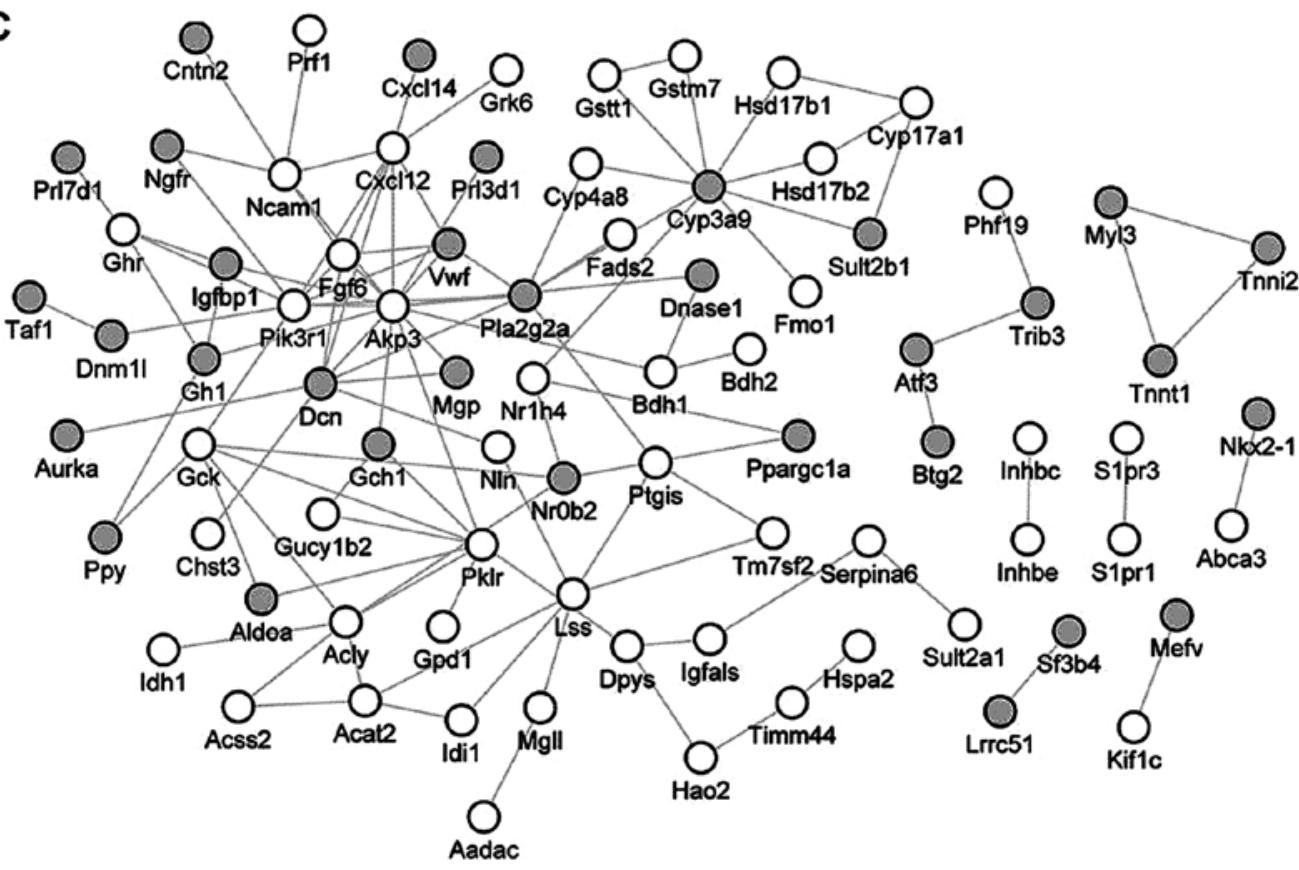

Figure 3. Protein-protein interaction network for the differentially expressed genes in the three comparison groups. (A) Sham-Sham vs. Sham-CLP (B) Sham-Sham vs. Burn-CLP. (C) Burn-CLP vs. Sham-CLP. CLP, cecum ligation and puncture. 
Table III. KEGG pathway enrichments with the downregulated DEGs in Sham-CLP and Burn-CLP compared with Sham-Sham and the downregulated DEGs in Burn-CLP compared with Sham-CLP.

\begin{tabular}{|c|c|c|c|}
\hline Groups & KEGG & P-value & Gene symbol \\
\hline \multirow[t]{10}{*}{ Sham-CLP vs. Sham-Sham } & $\begin{array}{l}\text { rno04080: Neuroactive } \\
\text { ligand-receptor interaction }\end{array}$ & $6.575 \times 10^{-4}$ & $\begin{array}{l}\text { Adrb2, Agtr1b, F2rl3, Gabrb1, } \\
\text { Galr3, Glra3, Glrb, Htr1a, } \\
\text { Lpar2, P2rx5, Pth2r, Tacr3 }\end{array}$ \\
\hline & rno04260: Cardiac muscle contraction & 0.004 & $\begin{array}{l}\text { Atpla4, Cacnb1, Cox6a2, Cox } 8 b \text {, } \\
\text { Fxyd2, Myl3 }\end{array}$ \\
\hline & rno05218: Melanoma & 0.013 & Fgf13, Fgf18, Fgf7, Mapk3, Pdgfa \\
\hline & rno04660: T cell receptor signaling pathway & 0.015 & $C d 28, C d 8 a, C d 8 b, I l 2, M a p k 3, T n f$ \\
\hline & rno04640: Hematopoietic cell lineage & 0.020 & $C d 2, C d 8 a, C d 8 b, I l 3, T n f$ \\
\hline & rno04010: MAPK signaling pathway & 0.025 & $\begin{array}{l}\text { Cacnb1, Fgf13, Fgf18, Fgf7, Hspall, } \\
\text { Mapk3, Pdgfa, Pla2g1b, Tnf }\end{array}$ \\
\hline & rno05330: Allograft rejection & 0.034 & $C d 28, I l 12 a, I l 2, T n f$ \\
\hline & rno04940: Type I diabetes mellitus & 0.047 & $C d 28, I l 12 a, I l 2, \operatorname{Tnf}$ \\
\hline & $\begin{array}{l}\text { rno04514: Cell adhesion } \\
\text { molecules (CAMs) }\end{array}$ & 0.048 & $C d 2, C d 28, C d 8 a, C d 8 b, M p z l 1, N r x n 3$ \\
\hline & $\begin{array}{l}\text { rno04060: Cytokine-cytokine } \\
\text { receptor interaction }\end{array}$ & 0.048 & $\begin{array}{l}\text { Agtrlb, Cx3cll, Ill2a, Il2, Il3, Pdgfa, } \\
\text { Tnf }\end{array}$ \\
\hline \multirow[t]{6}{*}{ Burn-CLP vs. Sham-Sham } & rno00650: Butanoate metabolism & $6.634 \times 10^{-4}$ & $\begin{array}{l}\text { Aldh3a2, Bdh1, Bdh2, Ehhadh, } \\
\text { Hmgcs2, Pdha2 }\end{array}$ \\
\hline & rno03320: PPAR signaling pathway & $8.298 \times 10^{-4}$ & $\begin{array}{l}\text { Acadm, Angptl4, Ehhadh, Fabp3, } \\
\text { Fabp7, Fads2, Gk, Hmgcs } 2\end{array}$ \\
\hline & rno00561: Glycerolipid metabolism & 0.003 & Aldh3a2, Dak, Gk, Lipc, Mgll, Pnliprp2 \\
\hline & rno00410: $\beta$-Alanine metabolism & 0.016 & Acadm, Aldh3a2, Dpys, Ehhadh \\
\hline & $\begin{array}{l}\text { rno00072: Synthesis and degradation } \\
\text { of ketone bodies }\end{array}$ & 0.015 & $B d h 1, B d h 2, H m g c s 2$ \\
\hline & rno00480: Glutathione metabolism & 0.023 & Gsta3, Gstm2, Gstt1, Idh1, Oplah \\
\hline \multirow[t]{3}{*}{ Burn-CLP vs. Sham-CLP } & $\begin{array}{l}\text { rno00072: Synthesis and degradation } \\
\text { of ketone bodies }\end{array}$ & 0.004 & Acat $2, B d h 1, B d h 2$ \\
\hline & rno00140: Steroid hormone biosynthesis & 0.011 & Cyp17a1, Hsd17b1, Hsd17b2, Sult2a2 \\
\hline & rno00650: Butanoate metabolism & 0.050 & Acat $2, B d h 1, B d h 2$ \\
\hline
\end{tabular}

DEGs, differentially expressed genes; CLP, cecum ligation and puncture.

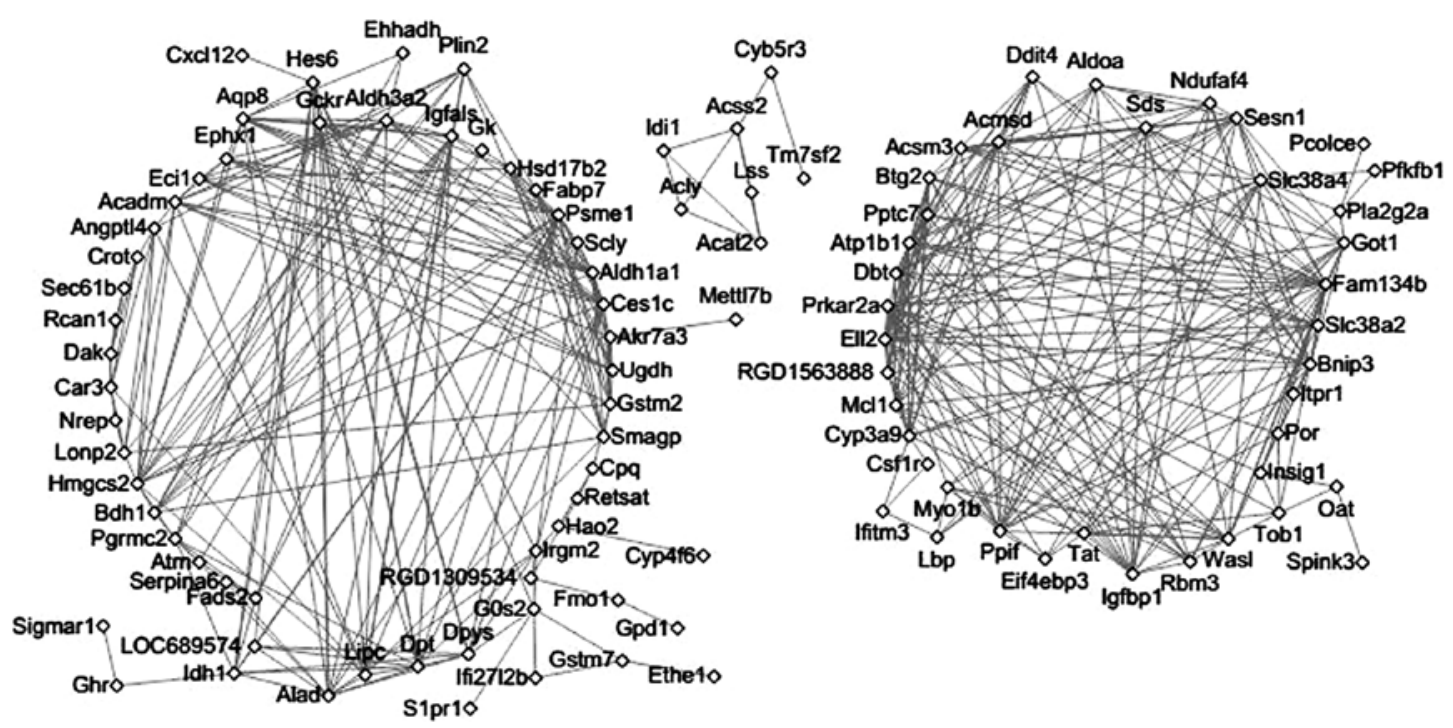

Figure 4. Co-expression network of the differentially expressed genes in Sham-Sham vs. Burn-CLP and Burn-CLP vs. Sham-CLP. CLP, cecum ligation and puncture. 
diverse electrophilic substrates and act as antioxidants against ROS (29). Accordingly, we hypothesized that Gsta3, Gstm2 and Gstt1 may be involved in sepsis through the pathway of glutathione metabolism.

In the present study, Ppargcla, which was upregulated in Burn-CLP compared with Sham-Sham, was screened as a transcription factor. However, in Sham-CLP, it was not differentially expressed, suggesting that Ppargcla could be associated with burn. AMPK-Ppargcla possibly contributes to autophagy activation leading to an antimicrobial response, which is a novel host defense mechanism (30). In the PPI network, Ppargcla could interact with Angptl4. Additionally, Angptl4 was significantly enriched in the PPAR signaling pathway. Angptl4 has been demonstrated to be involved with lipid metabolism, and the disorder of lipid metabolism is a vital issue in septic patients, particularly high-density lipoprotein, which protects against polymicrobe-induced sepsis in mice $(31,32)$. Consequently, we hypothesized that the interaction of Angptl4 and Ppargcla may be associated with sepsis through the PPAR signaling pathway.

In conclusion, Acadm, Ehhadh, Aqp8, Gsta3, Gstm2, Gstt1, Ppargcla and Angptl4 may be potential target genes for the treatment of burn sepsis. However, further studies are required to establish their mechanisms of action in burn sepsis.

\section{Acknowledgements}

The present study was supported by the Nosocomial Infection Control Research Fund of China (grant no. ZHYY 2014-0037), the Jinling Hospital Research Fund (grant no. YYMS 2014017), the Foundation of Medical Science and Technology Innovation (grant no. CNJ2014L004) and the Medical Innovation Fund (grant no. 14MS106).

\section{References}

1. Taylor FB Jr, Kinasewitz GT and Lupu F: Pathophysiology, staging and therapy of severe sepsis in baboon models. J Cell Mol Med 16: 672-682, 2012.

2. Martin GS, Mannino DM, Eaton S and Moss M: The epidemiology of sepsis in the United States from 1979 through 2000. N Engl J Med 348: 1546-1554, 2003.

3. Church D, Elsayed S, Reid O, Winston B and Lindsay R: Burn wound infections. Clin Microbiol Rev 19: 403-434, 2006.

4. Beffa DC, Fischman AJ, Fagan SP, Hamrahi VF, Paul KW, Kaneki M, Yu YM, Tompkins RG and Carter EA: Simvastatin treatment improves survival in a murine model of burn sepsis: Role of interleukin 6. Burns 37: 222-226, 2011.

5. Kraft R, Herndon DN, Finnerty CC, Cox RA, Song J and Jeschke MG: Predictive value of IL-8 for sepsis and severe infections after burn injury - A clinical study. Shock 43: 222-227, 2015.

6. Zu H, Li Q and Huang P: Expression of Treg subsets on intestinal $\mathrm{T}$ cell immunity and endotoxin translocation in porcine sepsis after severe burns. Cell Biochem Biophys 70: 1699-1704, 2014.

7. Banta S, Vemula M, Yokoyama T, Jayaraman A, Berthiaume F and Yarmush ML: Contribution of gene expression to metabolic fluxes in hypermetabolic livers induced through burn injury and cecal ligation and puncture in rats. Biotechnol Bioeng 97: 118-137, 2007

8. Afsari B, Geman D and Fertig EJ: Learning dysregulated pathways in cancers from differential variability analysis. Cancer Inform 13 (Suppl 5): 61-67, 2014.

9. Bolstad B: preprocessCore: A collection of pre-processing functions. R package version 1: 2013.

10. Huang W, Sherman BT and Lempicki RA: Systematic and integrative analysis of large gene lists using DAVID bioinformatics resources. Nat Protoc 4: 44-57, 2009.

11. Kanehisa M and Goto S: KEGG: Kyoto encyclopedia of genes and genomes. Nucleic Acids Res 28: 27-30, 2000.
12. Matys V, Fricke E, Geffers R, Gössling E, Haubrock M, Hehl R, Hornischer K, Karas D, Kel AE, Kel-Margoulis OV, et al: TRANSFAC: Transcriptional regulation, from patterns to profiles. Nucleic Acids Res 31: 374-378, 2003.

13. Franceschini A, Szklarczyk D, Frankild S, Kuhn M, Simonovic M, Roth A, Lin J, Minguez P, Bork P, von Mering C, et al: STRING v9.1: Protein-protein interaction networks, with increased coverage and integration. Nucleic Acids Res 41: D808-D815, 2013.

14. Saito R, Smoot ME, Ono K, Ruscheinski J, Wang PL, Lotia S, Pico AR, Bader GD and Ideker T: A travel guide to Cytoscape plugins. Nat Methods 9: 1069-1076, 2012.

15. Schmidt MV, Paulus P, Kuhn AM, Weigert A, Morbitzer V, Zacharowski K, Kempf VA, Brüne B and von Knethen A: Peroxisome proliferator-activated receptor $\gamma$-induced $\mathrm{T}$ cell apoptosis reduces survival during polymicrobial sepsis. Am J Respir Crit Care Med 184: 64-74, 2011.

16. Chiang MC, Cheng YC, Lin KH and Yen $\mathrm{CH}$ : PPAR $\gamma$ regulates the mitochondrial dysfunction in human neural stem cells with tumor necrosis factor alpha. Neuroscience 229: 118-129, 2013.

17. Singer M: The role of mitochondrial dysfunction in sepsis-induced multi-organ failure. Virulence 5: 66-72, 2014.

18. Kim JJ and Miura R: Acyl-CoA dehydrogenases and acyl-CoA oxidases. Structural basis for mechanistic similarities and differences. Eur J Biochem 271: 483-493, 2004.

19. Houten SM, Denis S, Argmann CA, Jia Y, Ferdinandusse S, Reddy JK and Wanders RJ: Peroxisomal L-bifunctional enzyme (Ehhadh) is essential for the production of medium-chain dicarboxylic acids. J Lipid Res 53: 1296-1303, 2012.

20. Hecker M, Sommer N, Voigtmann H, Pak O, Mohr A, Wolf M, Vadász I, Herold S, Weissmann N, Morty RE, et al: Impact of short-and medium-chain fatty acids on mitochondrial function in severe inflammation. JPEN J Parenter Enteral Nutr 38: 587-594, 2013.

21. Calamita G, Ferri D, Gena P, Liquori GE, Cavalier A, Thomas D and Svelto M: The inner mitochondrial membrane has aquaporin-8 water channels and is highly permeable to water. J Biol Chem 280: 17149-17153, 2005.

22. Wang JQ, Zhang L, Tao XG, Wei L, Liu B, Huang LL and Chen YG: Tetramethylpyrazine upregulates the aquaporin 8 expression of hepatocellular mitochondria in septic rats. J Surg Res 185: 286-293, 2013.

23. Schulte J, Struck J, Köhrle J and Müller B: Circulating levels of peroxiredoxin 4 as a novel biomarker of oxidative stress in patients with sepsis. Shock 35: 460-465, 2011.

24. Marchissio MJ, Francés DEA, Carnovale CE and Marinelli RA: Mitochondrial aquaporin-8 knockdown in human hepatoma HepG2 cells causes ROS-induced mitochondrial depolarization and loss of viability. Toxicol Appl Pharmacol 264: 246-254, 2012.

25. Banasik K, Justesen JM, Hornbak M, Krarup NT, Gjesing AP, Sandholt CH, Jensen TS, Grarup N, Andersson A, Jørgensen T, et al: Bioinformatics-driven identification and examination of candidate genes for non-alcoholic fatty liver disease. PLoS One 6: e16542, 2011.

26. Andrades MÉ, Morina A, Spasić S and Spasojević I: Bench-to-bedside review: sepsis - from the redox point of view. Crit Care 15: 230, 2011.

27. Dare AJ, Phillips AR, Hickey AJ, Mittal A, Loveday B, Thompson $\mathrm{N}$ and Windsor JA: A systematic review of experimental treatments for mitochondrial dysfunction in sepsis and multiple organ dysfunction syndrome. Free Radic Biol Med 47: 1517-1525, 2009.

28. Lowes DA and Galley HF: Mitochondrial protection by the thioredoxin-2 and glutathione systems in an in vitro endothelial model of sepsis. Biochem J 436: 123-132, 2011.

29. Ito M, Imai M, Muraki M, Miyado K, Qin J, Kyuwa S, Yoshikawa Y, Hosoi Y, Saito H and Takahashi Y: GSTT1 is upregulated by oxidative stress through p38-MK2 signaling pathway in human granulosa cells: Possible association with mitochondrial activity. Aging (Albany NY) 3: 1213-1223, 2011.

30. Yang CS, Kim JJ, Lee HM, Jin HS, Lee SH, Park JH, Kim SJ, Kim JM, Han YM, Lee MS, et al: The AMPK-PPARGC1A pathway is required for antimicrobial host defense through activation of autophagy. Autophagy 10: 785-802, 2014.

31. Hato T, Tabata M and Oike Y: The role of angiopoietin-like proteins in angiogenesis and metabolism. Trends Cardiovasc Med 18: 6-14, 2008.

32. Guo L, Ai J,Zheng Z, Howatt DA, Daugherty A, Huang B and Li XA: High density lipoprotein protects against polymicrobe-induced sepsis in mice. J Biol Chem 288: 17947-17953, 2013. 